\title{
VWA: ViewpointS Web Application to Assess Collective Knowledge Building
}

\author{
Philippe Lemoisson ${ }^{1,2(\bowtie)}$, Clarel M. H. Rakotondrahaja ${ }^{3,4}$, \\ Aroniaina Safidy Précieux Andriamialison ${ }^{4}$, Harish A. Sankar ${ }^{5}$, \\ and Stefano A. Cerri ${ }^{6,7}$ \\ ${ }^{1}$ CIRAD, UMR TETIS, 34398 Montpellier, France \\ philippe.lemoisson@cirad.fr \\ 2 TETIS, Univ Montpellier, AgroParisTech, CIRAD, CNRS, IRSTEA, \\ Montpellier, France \\ ${ }^{3}$ Ecole Doctorale de Modélisation Informatique, University of Fianarantsoa, \\ Fianarantsoa, Madagascar \\ ${ }^{4}$ Etech Research Lab, Arkeup Group, Antananarivo, Madagascar \\ \{c. rakotondrahaja, aroniaina\}@etechconsulting-mg.com \\ 5 Department of Computer Engineering, National Institute of Technology, \\ Kurukshetra, India \\ harishsa85@gmail.com \\ ${ }^{6}$ LIRMM, University of Montpellier \& CNRS, Montpellier, France \\ ${ }^{7}$ FBK: Fondazione Bruno Kessler, Trento, Italy \\ scerri@fbk.eu
}

\begin{abstract}
Collective intelligence is one major outcome of the digital revolution, but this outcome is hardly evaluated. By implementing a topological knowledge graph (KG) in the metaphor of a brain, the ViewpointS approach attempts to trace and assess the dynamics of collaborative knowledge building. Our approach relies on a bipartite graph of resources (agents, documents, topics) and time stamped "viewpoints" emitted by human or artificial agents. These viewpoints are typed (logical, mining, subjective). User agents feed the graph with resources and viewpoints and exploit maps where resources are linked by "synapses" aggregating the viewpoints. They reversely emit feedback viewpoints which tighten or loosen the synapses along the knowledge paths. Shared knowledge is continuously elicited against the individual "systems of values" along the agents' exploitation/feedback loops. This selection process implements a rudimentary form of collective intelligence, which we assess through innovative metrics.

In this paper, we present the exploitation/feedback loops in detail. We expose the mechanism underlying the reinforcement along the knowledge paths and introduce a new measure called Multi Paths Proximity inspired from the parallel neural circuits in the brain. Then we present the Web prototype VWA implementing the ViewpointS approach and set a small experiment assessing collective knowledge building on top of the exploitation/feedback loops.
\end{abstract}


Keywords: Collective intelligence $\cdot$ Knowledge graph $\cdot$ Knowledge map $\cdot$ Knowledge paths $\cdot$ Knowledge assessment $\cdot$ Reinforcement $\cdot$

Serendipitous learning $\cdot$ Unsupervised learning

\section{Introduction}

The so-called digital revolution has been progressively changing our lives in depth since the turn of the millennium, bringing in the hope of a collective intelligence [1]; the World Wide Web together with the internauts has often been compared to a collective brain.

Our previous work implements a topological numeric space where "knowledge shared within a community of agents is continuously elicited against the systems of values of the agents in a selection process." The goal of our approach is twofold: (i) to exploit the metaphor of the brain [2] for improving the collective construction of knowledge and (ii) to better exploit our digital traces in order to refine the understanding of our learning processes. We adopted a tripartite model 'resource/agent'-'resource'-'resource' called ViewpointS by storing and exploiting 'declarations by agents that two resources are close'. Assessing knowledge may seem a hazardous enterprise in such a context characterized by weak semantics. Whereas standard measurements exist in the case of explicit information retrieval, as listed in [3], none of those apply to serendipity: assessing informal learning remains an open issue despite the great deal of research work reviewed in [4]. Our approach is topology driven within the numeric space; it relies on a metric distance which opens the way both for "learning close to what we already know" in agreement with the principle of the "zones of proximal development" $[5,6]$ and for assessing knowledge acquisition.

This paper is a step forward in deepening the brain metaphor exposed by Edelman in [7]; we implement a feedback mechanism underlying the reinforcement along the knowledge paths and design a new metric taking into account the multiplicity of the knowledge paths.

We start in Sect. 2 by recalling the ViewpointS paradigm, presenting the feedback mechanism and defining a new metric called Multi Paths Distance. In Sect. 3 we briefly present the ViewpointS Web Application implementing the approach. In Sect. 4 we demonstrate the assessment of collective knowledge building through a small experiment using the feedback mechanism. Section 5 starts with a discussion about the results and then presents short term perspectives.

\section{The ViewpointS Paradigm}

The ViewpointS paradigm previously presented in [8-10] builds up upon trust towards 'peers', would they be humans, databases or mining algorithms. A community of human agents combines in a fully transparent and trackable way their own knowledge and feelings with declarations extracted from the Web. This happens in one single numeric space where: 
(i) Inferences of the semantic Web (e.g. "Marguerite Yourcenar was born in 1903") as well as 'objective' declarations of the users (e.g. "I was born in Paris") provide the Logical viewpoints;

(ii) Statistical recommendations due to mining algorithms provide the Mining viewpoints (e.g. "https://www.aps.org/publications/apsnews/200207/history.cfm is related to "serendipity"');

(iii) Spontaneous opinions, feelings and feedbacks of the community of agents provide the Subjective viewpoints (e.g. "I like this book"; "I think John is the person to contact if you are interested in "Serendipity"),

The formalism adopted in the ViewpointS paradigm is briefly recalled in Sect. 2.1; for more details the reader might refer to $[8,9]$. The feedback mechanism is presented in Sect. 2.2. The metrics governing the exploration of knowledge are presented in Sect. 2.3.

\subsection{Interconnected Observation/Action Loops in a Numeric Knowledge Space}

In the ViewpointS paradigm, agents, documents and topics constitute the knowledge resources; those are interlinked via the viewpoints. The viewpoint $\left(\mathrm{r}_{1},\left\{\mathrm{r}_{2}, \mathrm{r}_{3}\right\}, \theta, \tau\right)$ stands for: 'agent $r_{1}$ ' (human or artificial) declares at time ' $\tau$ ' that ' $r_{2}$ ' and ' $r_{3}$ ' are connected in the paradigm $\theta$ (Logical versus Mining versus Subjective).

We call Knowledge Graph the bipartite graph consisting of knowledge resources and viewpoints imported or emitted by the users themselves or by artificial agents. Given two knowledge resources, the aggregation of all the viewpoints interlinking them is called a synapse; it can be quantified and interpreted in terms of proximity by choosing a perspective. This is illustrated in Fig. 1.

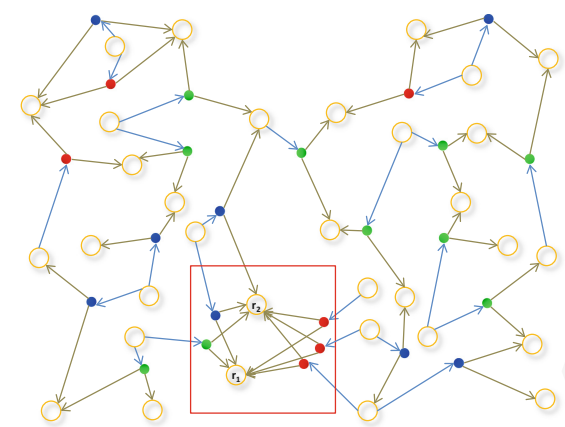

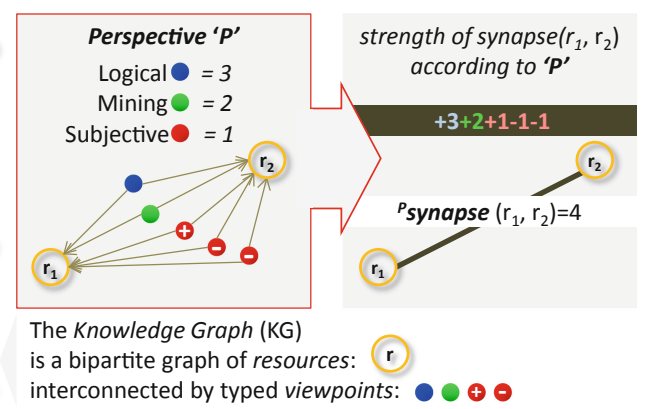

Fig. 1. The bipartite graph of resources and viewpoints is exploited by the user after choosing a perspective ruling the aggregation of viewpoints

The left part of Fig. 1 shows the bipartite graph of resources and viewpoints. When zooming on the two resources $r_{1}$ and $r_{2}$, we see that five viewpoints (emitted by five distinct agents) interconnect them. These five viewpoints are colored according to their types: blue $=$ Logical, green $=$ Mining and red = Subjective . Subjective viewpoints have a polarity: in the example, one is 'positive' (declaring proximity according to the subjectivity of the emitter) whereas two are 'negative' (denying proximity). 
By choosing a perspective, any agent can tune the rules aggregating the qualitative viewpoints into quantitative synapses according to his own preferences with respect to the extraction of the knowledge available on the graph. These rules can integrate any type of IF-THEN predicate filtering the time-stamp, the emitter or the type of the viewpoints. In the examples of this paper, choosing the perspective simply consists in tuning the respective weights of the Logical, Mining and Subjective viewpoints, e.g. in Fig. 1 the chosen perspective ' $\mathrm{P}$ ' consists in attributing the weights 3, 2 and 1 respectively for the Logical, Mining and Subjective. As a result, ${ }^{P}$ synapse $\left(\mathrm{r}_{1}, \mathrm{r}_{2}\right)=+3+2+1-1-1=4$, which is interpreted as "the proximity between $r_{1}$ and $r_{2}$ is " 4 "" OR " $r_{1}$ and $r_{2}$ are at distance $1 / 4$ ".

Given a perspective ' $P$ ', we call ${ }^{\mathrm{P}} \mathrm{KM}$ (knowledge map according to ' $P$ ') the following undirected labelled graph:

- the nodes of ${ }^{\mathrm{P}} \mathrm{KM}$ are the resources of $\mathrm{KG}$;

- the edges of ${ }^{\mathrm{P}} \mathrm{KM}$ are the positive ${ }^{P}$ synapses labelled by their values.

The shared semantics emerge from the dynamics of the intricate observation/action loops among the community of agents interacting with the $\mathrm{KG}$ through the ${ }^{\mathrm{P}} \mathrm{KM}$. Agents choose a perspective, browse the knowledge map ${ }^{\mathrm{P}} \mathrm{KM}$ and exploit the shared knowledge issued from the viewpoints of the whole community (observation), and reversely update the knowledge graph $\mathrm{KG}$ by adding new viewpoints expressing their feedback (action). Intertwining exploitation of shared resources and feedback on their links enhances collaborative knowledge building; this has been analyzed in [11] and illustrated in [12]. In the ViewpointS paradigm, the shared knowledge is continuously elicited against the individual systems of values of the members of the community in a selection process.

\subsection{Reinforcing Versus Weakening Knowledge Paths in the Loops}

Subjective viewpoints have a polarity. Positive (resp. negative) viewpoints are used for declaring (resp. denying) proximity between resources. Through subjective viewpoints, an agent may create direct connections: (i) between him/her and a given resource, e.g. "like/dislike" or (ii) between two given resources, e.g. "match/mismatch".

A complementary mechanism involves a special category of subjective viewpoints called feedback viewpoints; it is illustrated in this paper. Feedback viewpoints allow agents to strengthen oR weaken the shortest path ${ }^{1}$ between two distant resources, by distributing fragments of a subjective viewpoint along the shortest path in the metaphor of the reinforcement of synapses in our neural circuits. We illustrate this by an imaginary use case based on the following KG:

- documents have been connected via Mining viewpoints to topics, e.g. 'saying92' has been connected to 'topic-A';

- human agents (Marguerite Yourcenar, Voltaire...) have been connected via Logical viewpoints to topics (topic-G, topic-S ...);

- by transitivity, the agents are connected to the documents by paths where the proximities are computable.

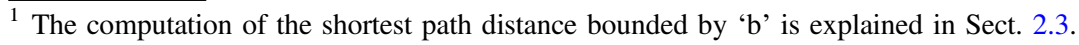


Three human agents $\left(\mathrm{HA}_{1}, \mathrm{HA}_{2}\right.$ and $\left.\mathrm{HA}_{3}\right)$ exploit the knowledge through the perspective $\mathrm{P}_{0}$ where the weights of the Logical, Mining and Subjective viewpoints are all equal to ' 1 '; the corresponding knowledge map is denoted ${ }^{\mathrm{P} 0} \mathrm{KM}$. They react to the proximities by emitting the Subjective feedback viewpoints illustrated in Fig. 2.

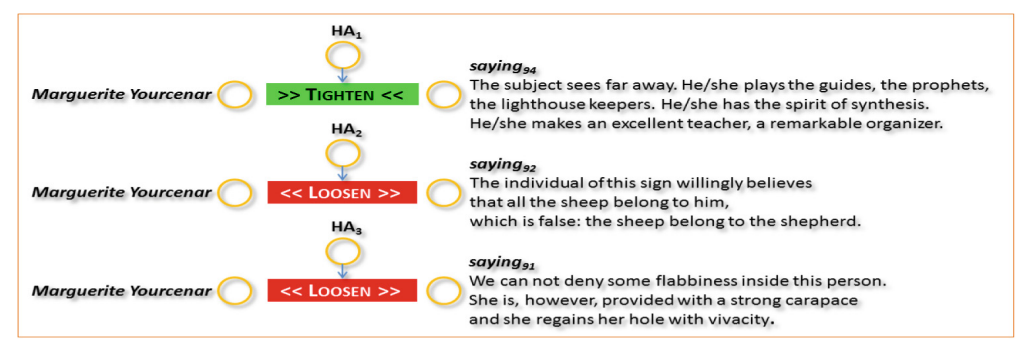

Fig. 2. The human agents $\mathrm{HA}_{1}, \mathrm{HA}_{2}$ and $\mathrm{HA}_{3}$ update the proximities between Marguerite Yourcenar and saying ${ }_{94}$ saying $_{92}$ and saying $_{91}$ respectively.

Figure $3 \mathrm{~A}$ below illustrates the initial state of ${ }^{\mathrm{P} 0} \mathrm{KM}$. The Subjective feedback viewpoints yield the final state illustrated in Fig. 3B.

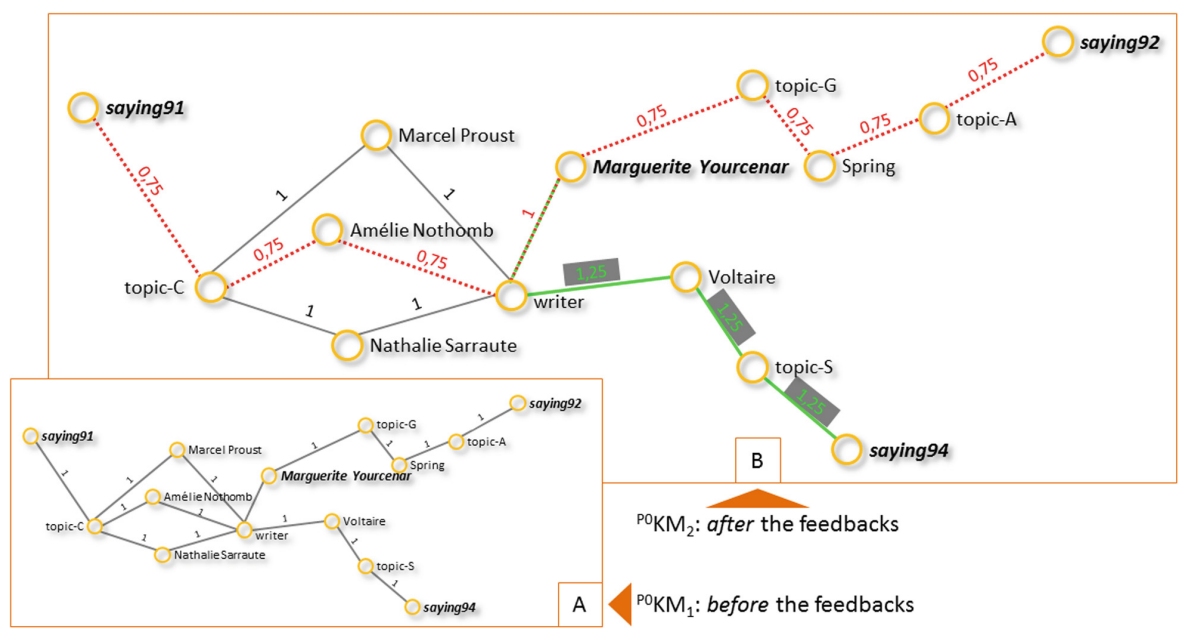

Fig. 3. Evolution $\mathrm{A} \rightarrow \mathrm{B}$ of the ${ }^{\mathrm{P} 0} \mathrm{KM}$ as a consequence of the TIGHTENING and LOOSENING; the labels are the strengths of the synapses (Color figure online)

- The shortest path between Marguerite Yourcenar and saying ${ }_{94}$ counts ' $\mathrm{n}=4$ ' edges and goes through 'writer', 'Voltaire' and 'topic-S'. HA 1 TIGHTENS the path through a positive feedback viewpoint by adding a subjective viewpoint of ' $+1 /(\mathrm{n}=4)$ ' on each edge; each corresponding ${ }^{P}$ synapse evolves from ' 1 ' (grey lines in image A) to ' 1,25 ' (green lines in image B). 
- The shortest path between Marguerite Yourcenar and saying ${ }_{91}$ counts '4' edges and goes through 'topic-G', 'spring' and 'topic-A'. HA 2 LOOSENS the path through a negative feedback viewpoint by adding a subjective viewpoint of ' $-1 / 4$ ' on each edge; each corresponding ${ }^{P}$ synapse evolves from ' 1 ' (grey lines in A) to '0,75' (red dotted lines in B).

- The shortest path between Marguerite Yourcenar and saying ${ }_{91}$ counts '4' edges and goes through 'writer', 'Amélie Nothomb' and 'topic-C'. $\mathrm{HA}_{3}$ LOOSENS the path through a negative feedback viewpoint by adding in the $\mathrm{KG}$ a subjective viewpoint of ' $-1 / 4$ ' on each edge; each corresponding ${ }^{P}$ synapse evolves from ' 1 ' (grey lines in A) to ' 0,75 ' (red dotted lines in B).

\subsection{The Metrics Sustaining the Exploitation of Knowledge}

In our previous work [9], the metric in use was the "shortest path distance bounded by 'b"', denoted 'SPD, an adaptation of Dijkstra's algorithm with acceptable complexity ${ }^{2}$ which: (i) scans all the paths respecting the threshold ' $b$ ' between two given resources and (ii) computes the shortest path. Given a ${ }^{\mathrm{P}} \mathrm{KM}$, the length of an edge $r_{i}-r_{j}$ is defined as $1 /^{P}$ synapse $\left(r_{i}, r_{j}\right)$; the length of a path $r_{i}-\ldots-r_{j} \ldots-r_{k}$ is the sum of the edges' lengths. ' $\mathrm{SPD}\left(\mathrm{r}_{\mathrm{i}}, \mathrm{r}_{\mathrm{j}}\right)$ returns 'NA' if no path of length $<=b$ link $r_{i}$ to $r_{j}$; it returns $\mathrm{d}<=\mathrm{b}$ otherwise. ${ }^{\mathrm{b}}$ SPD has been illustrated in the imaginary case developed in [8].

In this paper, we wish to consider multiple parallel paths when computing proximities in $\mathrm{P}$ KM. We characterize an "equivalent synapse" expressing a proximity between two given resources by taking into account all the possible paths; we call it "multi paths proximity bounded by ' $b$ "” and denote it ${ }^{b}$ MPP. The computation of ${ }^{b}$ MPP requires two preliminary definitions:

- we call ${ }^{\mathrm{b}}$ Neighbours $\left(\mathrm{r}_{0}\right)$ the set of resources $\mathrm{r}_{\mathrm{x}}$ such that ${ }^{\mathrm{b}} \operatorname{SPD}\left(\mathrm{r}_{0}, \mathrm{r}_{\mathrm{x}}\right)$ is available;

- we call ${ }^{\mathrm{b}}$ Paths $\left(\mathrm{r}_{0}, \mathrm{r}_{\mathrm{x}}\right)$ the set of all paths $\left(\mathrm{r}_{0}, \ldots, \mathrm{r}_{\mathrm{x}}\right)$ of length $<=\mathrm{b}$ between $\mathrm{r}_{0}$ and resources belonging to ${ }^{\mathrm{b}}$ Neighbours $\left(\mathrm{r}_{0}\right)$.

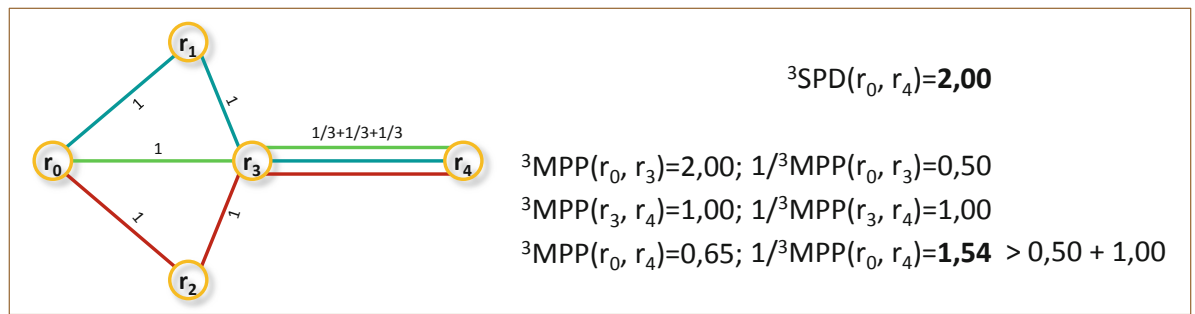

Fig. 4. Computation of ${ }^{3} \mathrm{MPP}\left(\mathrm{r}_{0}, \mathrm{r}_{4}\right)$. The label " $1 / 3+1 / 3+1 / 3$ " between $r_{3}$ and $r_{4}$ indicates that synapse $\left(r_{3}, r_{4}\right)$ participates to three paths linking $r_{3}$ to $r_{4}$, each of these paths using a channel capacity of $1 / 3$ of the strength of the synapse.

\footnotetext{
The worst case complexity of Dijkstra's algorithm is $\mathrm{O}\left(\mathrm{N}_{\mathrm{W}}{ }^{2} \mathrm{~N}_{\mathrm{R}}{ }^{2}\right)$, where $\mathrm{N}_{\mathrm{W}}$ is the number of viewpoints in $K G$ and $N_{R}$ is the number of resources in KG. In ${ }^{b} S P D$, the worst case complexity is practically never reached because of the bound ' $\mathrm{b}$ '.
} 
The computation of ${ }^{b}$ MPP $\left(r_{1}, r_{2}\right)$ illustrated in Fig. 4 obeys four rules:

- rule 1: given ' $\mathrm{b}$ ', we restrict the computation to the following subgraph of $\mathrm{KG}$ : ${ }^{\mathrm{b}} \mathrm{Paths}\left(\mathrm{r}_{1}, \mathrm{r}_{2}\right)$;

- rule 2: when ' $\mathrm{n}$ ' paths of ${ }^{\mathrm{b}} \mathrm{Paths}\left(\mathrm{r}_{1}, \mathrm{r}_{2}\right)$ share a ${ }^{P}$ synapse, we consider a channel capacity 'cc $={ }^{P}$ synapse/n' for each path, and a pseudo-length ' $\mathrm{L}_{\mathrm{edge}}=1 / \mathrm{cc}$ ' for this edge in each path;

- rule 3: for each path $\mathrm{i}_{\mathrm{i}}$ between $\mathrm{r}_{1}$ and $\mathrm{r}_{2}$, we consider the pseudo-length $\overline{\mathrm{LL}}\left(\right.$ path $\left._{\mathrm{i}}\right)=\sum$ edges in path $\mathrm{L}_{\text {edge }}\left(\right.$ path $\left.\left._{\mathrm{i}}\right)\right)$;

- rule 4: ${ }^{\mathrm{b}} \mathrm{MPP}\left(\mathrm{r}_{1}, \mathrm{r}_{2}\right)=\sum_{\mathrm{i}=1, \mathrm{n}} 1 / \mathrm{LL}\left(\mathrm{path}_{\mathrm{i}}\right)$.

$1 / /^{\mathrm{b}} \mathrm{MPP}\left(\mathrm{r}_{0}, \mathrm{r}_{0}\right)=0.1 /^{\mathrm{b}} \mathrm{MPP}$ satisfies the symmetry condition: $1 /^{\mathrm{b}} \mathrm{MPP}\left(\mathrm{r}_{1}\right.$, $\left.\mathrm{r}_{2}\right)=1 /{ }^{\mathrm{b}} \mathrm{MPP}\left(\mathrm{r}_{2}, \mathrm{r}_{1}\right)$. However $1 /^{\mathrm{b}} \mathrm{MPP}$ DOES NOT satisfy the triangle inequality: $1 /^{\mathrm{b}} \mathrm{MPP}$ $\left(\mathrm{r}_{0}, \mathrm{r}_{4}\right)>1 /^{\mathrm{b}} \mathrm{MPP}\left(\mathrm{r}_{0}, \mathrm{r}_{3}\right)+1 /^{\mathrm{b}} \mathrm{MPP}\left(\mathrm{r}_{3}, \mathrm{r}_{4}\right)$. It is NOT a metric distance.

\section{VWA, the ViewpointS Web Application}

The ViewpointS Web Application (VWA) has been implemented in the Spring Web MVC framework providing Model-View-Controller (MVC) architecture; the KG is stored in a Postgresql database exploited through a Java API. Once logged in, any user can create resources and emit viewpoints shared by the community.

The web based graphical user interface presents 7 significant zones marked in red in Fig. 5 below.

Zone 1 lists the menu commands:

- New resource: Knowledge graphs (KGs) are populated with different classes of resources: "artificial agent", "human agent", "numeric document", "physical document", or "topic". Every resource class has a distinct color when appearing in the draw area (Zone 3). Only in case of a Numeric document, an additional option of attaching (uploading) an URL is presented. All resources are identified based on their name. It must be noted that the set of resources is shared by all the KGs; on the contrary, each viewpoints belongs to one and only one KG.

- New Logical Viewpoint: Two resources are to be selected before a logical viewpoint can be emitted (by the current user) to interconnect them. A radio-button (containing ' + ' sign) is preselected.

- New Subjective Viewpoint: Similar to the previous option, two resources are to be mentioned. Four radio buttons allow the user to tune the polarity and strength of the emitted viewpoint. Choosing '-', '0', '+' and '++' signs create a 'negative', 'neutral', 'positive' and 'double positive' viewpoint respectively.

- New Feedback: A feedback between two resources can either be positive (TIGHTEN) or negative (LOOSEN) as depicted by the '-' and ' + ' signs respectively ${ }^{3}$.

\footnotetext{
${ }^{3}$ In either case, the result is the emission of a series of fragmented viewpoints along the shortest path between the two resources, as explained in Sect. 2.2.
} 


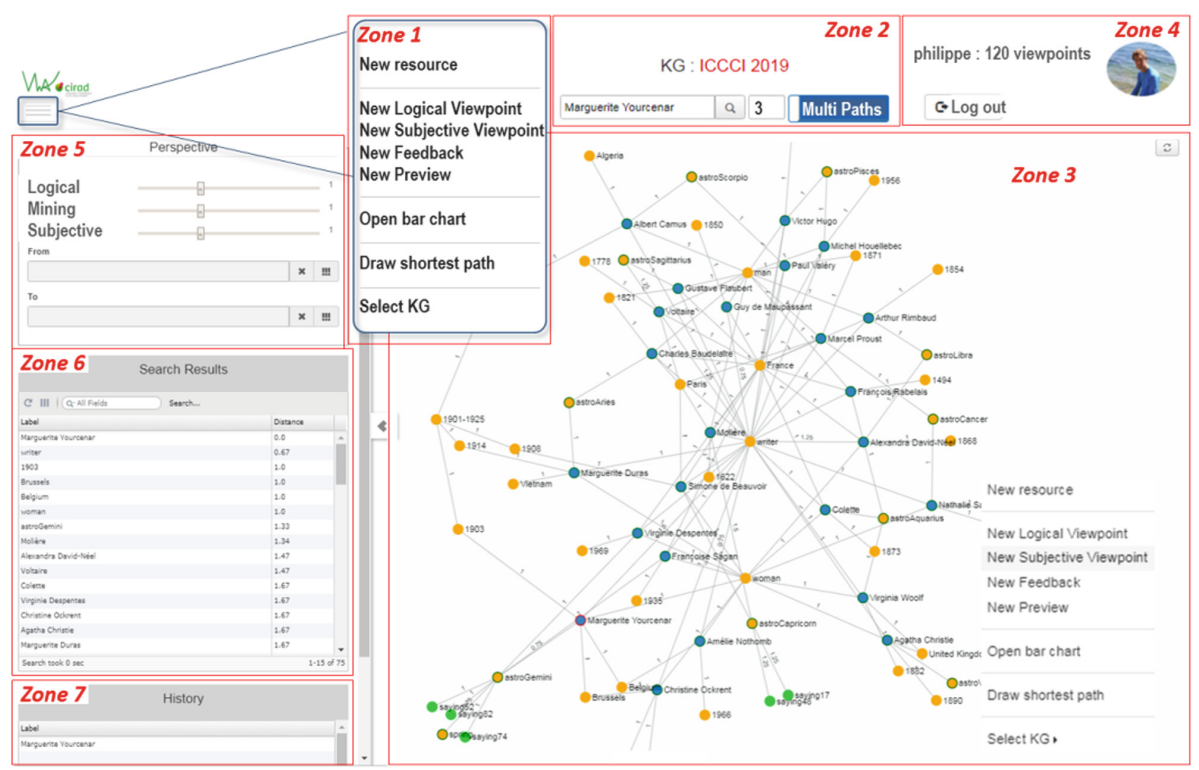

Fig. 5. The graphical user interface of VWA as it appears after user authentication and log in (Color figure online)

- New Preview: A resource ' $\mathrm{x}$ ' can be connected to a Numeric document that will appear on right click on ' $\mathrm{x}$ ' in the draw area. Nota: preview viewpoints do not contribute to the synapses.

- Open bar chart: This function calculates all the proximities (according to the metrics selected in zone 2) between (i) a given resource ' $x$ ' and (ii) a vector ' $Z$ ' of selected resources playing the role of referential. The result is a bar chart drawing the $\mathrm{Z}$ profile of ' $\mathrm{x}$ '.

- Draw shortest path: Operates on two resources selected from "Search results" (Zone 6) and/or "History" (Zone 7). The shortest path between them according to the current perspective is computed and drawn in the draw area (Zone 3).

- Select KG: A list of knowledge graphs (created by the administrator) is provided to the user.

Zone 2 is the area for activating a search, on the basis of the parameters defined in the perspective (Zone 5). The results are shown graphically in the draw area (Zone 3) and also listed in "search results" (Zone 6). The search is conducted on the basis of resource name (auto completion is available). The neighborhood radius (' $b$ ' for ${ }^{b} \mathrm{SPD}$ and ${ }^{b}$ MPP as mentioned in Sect. 2.2) tunes the depth of the search. A toggle between "shortest-path" and "multi-paths" metrics is available".

Zone 3 is the draw area where the neighborhood of the target in ${ }^{\mathrm{P}} \mathrm{KM}$ is edited with resources interlinked by 'synapses' with labels corresponding to their weights.

\footnotetext{
${ }^{4}$ These metrics have been described in Sect. 2.2 .
} 
The target of the search is circled in red. The graph can be resized and reshaped with simple mouse drag operations. Right clicking on a resource circled in green shows its preview in a tiny dialogue box. They can be further elaborated with a left click. The edition is cumulative until a change of perspective or a "clean visu" (up-right button)

Zone 4 is self-explanatory as shown in the Fig. 5. The picture of the agent and the number of viewpoints emitted by the agent are displayed. The logout button is also present in this zone.

Zone 5 is the perspective pane that monitors the aggregation of viewpoints into synapses as explained in Sect. 2.1. In the current state of the prototype, only simple tuning of perspectives is available: the synapses of the knowledge graph can be revaluated according to the three sliders corresponding to the viewpoints types (Logical, Mining and Subjective). Besides, viewpoints can be filtered according to time ' $\tau$ '.

Zone 6 contains the search results. These results are ranked according to their proximities from the selected resource. Double clicking on a resource can select the resource and copy it in the search area.

Zone 7 keeps track of every resource that has been successfully searched and selected.

The ViewpointS Web Application (VWA) is regularly enriched with new functionalities; a demo version, where the sessions are not persistent, is available at url: viewpoints.cirad.fr/vwademo.

\section{Assessing Collective Knowledge}

In order to assess the collective knowledge with the metrics presented in Sect. 2.3, we have set a toy experiment where three VWA users express knowledge about a panel of writers. The knowledge graph (KG) is initially populated by Logical viewpoints and Mining viewpoints; the three VWA users express their own opinions about the writers by adding subjective feedback viewpoints. Then we use the metrics presented in Sect. 2.3 to measure the reinforcement versus weakening of the knowledge paths.

\subsection{Settings}

The following resources initially populate the KG:

- ' $\mathrm{HA}_{1}$ ', ' $\mathrm{HA}_{2}$ ' and ' $\mathrm{HA}_{3}$ ': human agents sources of the Subjective knowledge in VWA; other "human agents", e.g. 'Marguerite Yourcenar'

- 'Authors Database': artificial agent source of the Logical knowledge

- 'Astrological Statistics': artificial agent source of the Mining knowledge

- a few "topics": 'solar year', 'spring', 'astroCancer', 'astroSagittarius', ...

- numeric documents called "sayings" that the users may read by right-clicking on them in the KM, e.g. 'saying ${ }_{81}$ : The person does not look into her past: she dives into it, she bathes there. She does not make plans for the future; she makes plans for the past.' 
The following viewpoints initially populate the $\mathrm{KG}$ :

- Logical viewpoints emitted by 'Authors DATABASE' interlink the writers, their astrological signs and the seasons of the solar year, e.g. 'Marguerite Yourcenar LINKEDTo astroGemini'

- Mining viewpoints emitted by 'Astrological Statistics' interlink the sayings and

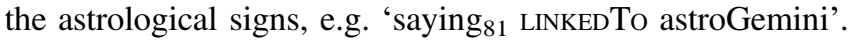

Thethreeusers' $\mathrm{HA}_{1}$ ', 'HA${ }_{2}$ 'and' $\mathrm{HA}_{3}$ 'areinvitedtobrowsetheknowledgegraphunderthe perspective $\mathrm{P}_{1}$ illustratedinFig. 6 .Theybenefitinrealtimefromthecontributionsoftheothers BUT are deprived from the Mining viewpoints, i.e. they are not influenced by the knowledge of 'AstrologicalStatistics'. During thisbrowsing, theyareinvited toexpressinfeedback their ownopinionsabouttheproximitiesbetweenthe"sayings"andthewritersbyexclusivelyusing the TIGHTENandLOOSENfacilitiesexposedinSect. 2.2.

\subsection{Results}

The first result is a proof of concept: we have implemented the mechanism described in Sect. 2.2 and proven the reinforcement versus weakening of some knowledge paths: Fig. 6 (directly issued from VWA) is a perfect match of Fig. 3 (drawn at design phase) through the following translations: topic-A $\rightarrow$ astroAries; topic-C $\rightarrow$ astroCancer; topic-G $\rightarrow$ astroGemini; topic-S $\rightarrow$ astroSagittarius. After the experiment, the probability to reach saying ${ }_{94}$ from Marguerite Yourcenar when browsing along the paths has become stronger than the probability to reach saying ${ }_{91}$ or saying $_{92}$; in other words, the communities of agents have collectively declared that saying 94 fits her better than by the two other sayings.

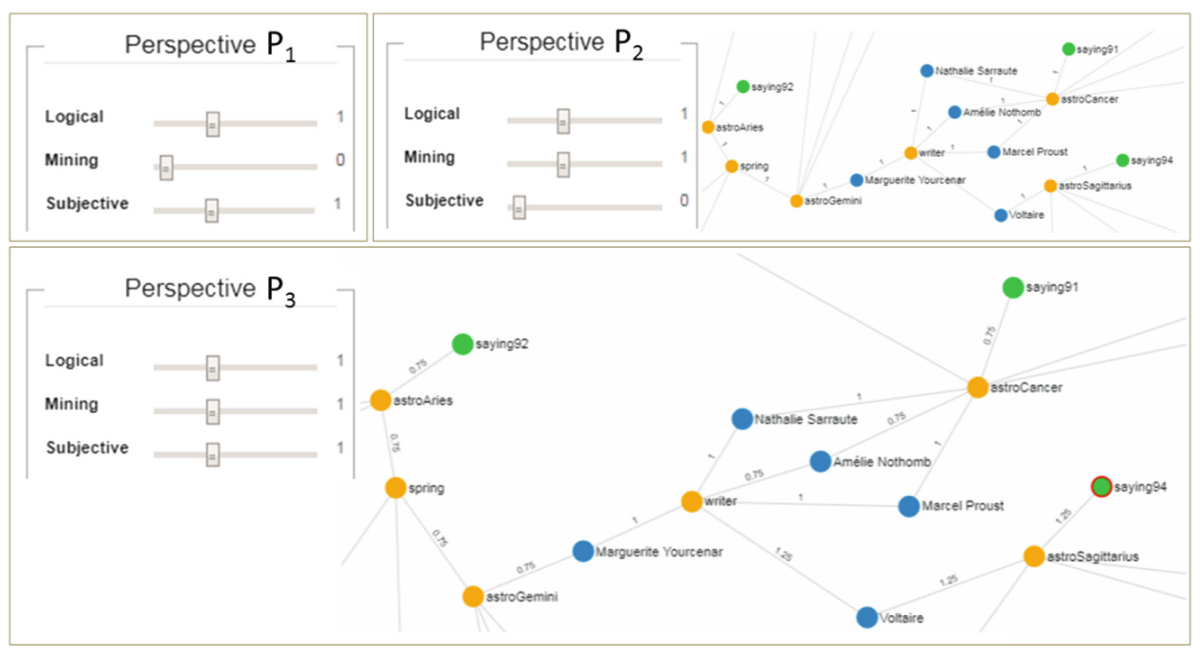

Fig. 6. Perspectives and corresponding knowledge maps: $\mathrm{P}_{1}$ considers Logical+Subjective viewpoints, $\mathrm{P}_{2}$ considers Logical+Mining and $\mathrm{P}_{3}$ considers Logical+Mining+Subjective

The results presented in Fig. 7 illustrate a search where the target is 'Marguerite Yourcenar'. 


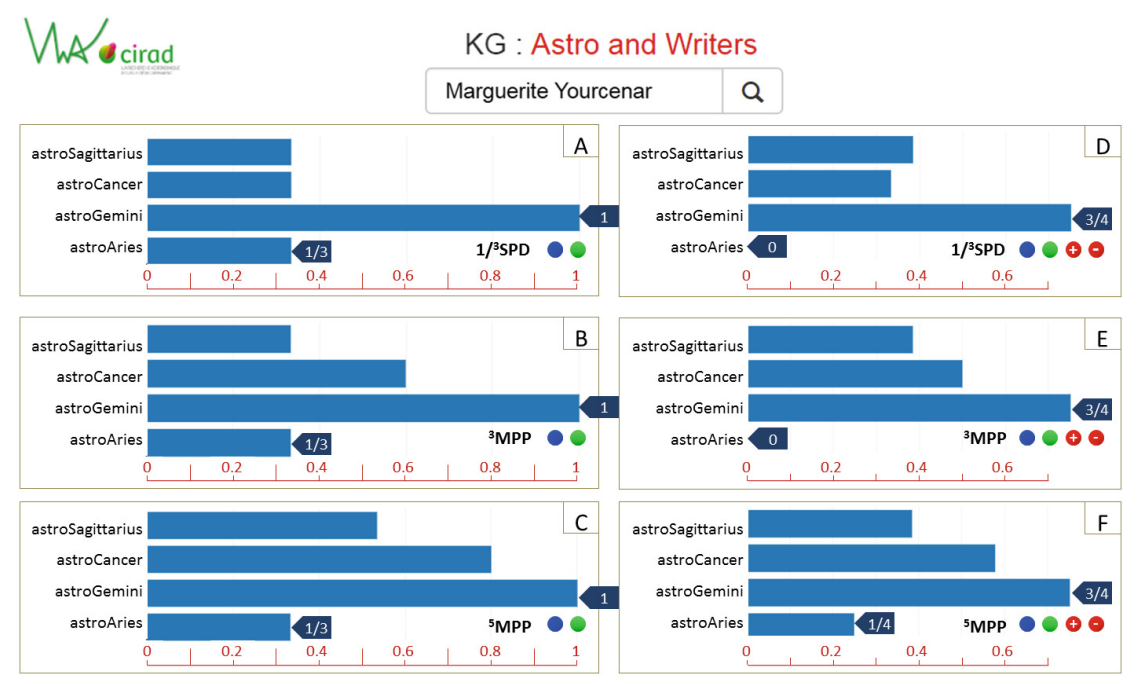

Fig. 7. Impact of the feedbacks on the "astrological profile" of Marguerite Yourcenar (M. Y.); the bar charts reflect proximities according to two distinct perspectives, "WITH subjective viewpoints' in the right diagrams) and various metrics (1/SPD versus MPP) (Color figure online)

By comparing left to right we assess the reinforcement mechanism. The left diagrams $\mathrm{A}, \mathrm{B}, \mathrm{C}$ give proximities according to perspective $\mathrm{P}_{2}$ : WITHOUT subjective viewpoints (only blue and green viewpoints), i.e. with all viewpoints BEFORE the feedback by the users. The right diagrams D, E, F give proximities according to perspective $\mathrm{P}_{3}$ : WITH the subjective viewpoints, i.e. with all viewpoints INCLUDING the feedback by the users (blue, green, red+ and red- viewpoints).

By comparing up to down, we compare the metrics: A\&D use the shortest path proximity $1 /{ }^{3} \mathrm{SPD}$; B\&C use the multi paths proximity ${ }^{3} \mathrm{MPP}$; E $\& \mathrm{~F}$ use the multi paths proximity ${ }^{5} \mathrm{MPP}$.

The measures displayed in the six diagrams open to the following comments:

- given a perspective (either $\mathrm{P}_{2}$ or $\mathrm{P}_{3}$ ), proximities computed by MPP are equal or bigger than proximities computed by 1/SPD: the proximities in ' $\mathrm{B}$ ' are bigger than in ' $A$ '; the proximities in ' $E$ ' are bigger than in ' $D$ '.

- 'MPP increases when ' $b$ ' increases: the proximities in ' $C$ ' are bigger than in ' $\mathrm{B}$ '; the proximities in ' $F$ ' are bigger than in ' $E$ '.

- both TIGHTEN and LOOSEN operate independently from the metric or the bound ' $b$ ' chosen: by comparing successively $A \& D, B \& C$ and $E \& F$, we can see that the respective proximity between: (i) 'M. Y.' and 'astroAries' decreases, (ii) 'M. Y.' and 'astroGemini' decreases, (iii) 'M. Y.' and 'astroCancer' decreases, (iv) 'M. Y.' and 'astroSagittarius' increases. 


\section{Discussion}

In this paper, we have put the focus on the exploitation/feedback loops of a community of agents sharing knowledge resources. We have provided features and metrics aimed at aggregating individual viewpoints into measurable proximities and presented the ViewpointS Web Application (VWA), working online.

We have proved the concept of a feedback mechanism impacting the topology of the knowledge graph in the metaphor of a collective brain by setting an experiment where users were specifically required to feedback. We have described and operated a new measure called "multi paths proximity (MPP)". MPP does not respect the triangle inequality. MPP yields greater proximities than 1/SPD and these proximities increase with the bound ' $b$ ' reflecting the depth of the search. We have opened the way to the assessment of collective knowledge: (i) perspective-dependent knowledge paths appear through Knowledge Maps and (ii) these paths are strengthened or weakened depending on the dynamics of the interactions.

Our next step will consist in setting a more ambitious real-life experiment in order to better analyze MPP in comparison with $1 / \mathrm{SPD}$, especially by comparing their respective stabilities along the dynamics of the interactions, and better explicit the benefits driven from the brain metaphor in assessing collective knowledge.

\section{References}

1. Gruber, T.: Collective knowledge systems: where the Social Web meets the Semantic Web. Web. Semant. Sci. Serv. Agents World Wide Web 6(1), 4-13 (2008)

2. Cerri, S.A., Lemoisson, P.: Tracing and enhancing serendipitous learning with ViewpointS. In: Frasson, C., Kostopoulos, G. (eds.) Brain Function Assessment in Learning. LNCS (LNAI), vol. 10512, pp. 36-47. Springer, Cham (2017). https://doi.org/10.1007/978-3-31967615-9_3

3. Qin, T., Liu, T.-Y., Xu, J., Li, H.: LETOR: a benchmark collection for research on learning to rank for information retrieval. Inf. Retr. 13(4), 346-374 (2010)

4. Sefton-Green, J.: Literature Review in Informal Learning with Technology Outside School. A NESTA Futurelab Series - report 7 (2004)

5. Piaget, J.: Development and learning. In: Piaget Rediscovered (1964)

6. Vygotsky, L.S.: Mind in Society: The Development of Higher Psychological Processes. Harvard University Press, Cambridge (1980)

7. Edelman, G.M.: Neural Darwinism: The Theory of Neuronal Group Selection. Basic Books, New York (1989)

8. Lemoisson, P., Cerri, S.A.: ViewpointS: towards a collective brain. In: Nguyen, N.T., Pimenidis, E., Khan, Z., Trawiński, B. (eds.) ICCCI 2018. LNCS (LNAI), vol. 11055, pp. $3-$ 12. Springer, Cham (2018). https://doi.org/10.1007/978-3-319-98443-8_1

9. Lemoisson, P., Surroca, G., Jonquet, C., Cerri, S.A.: ViewPointS: capturing formal data and informal contributions into an evolutionary knowledge graph. Int. J. Knowl. Learn. 12(2), 119-145 (2018) 
10. Lemoisson, P., Surroca, G., Jonquet, C., Méric, L., Cerri, S.A.: ViewPointS: bridging the gap between explicit knowledge of the semantic Web and implicit knowledge of the social web. Semant. Web J. Special Issue

11. Laurillard, D.: A conversational framework for individual learning applied to the 'learning organisation' and the 'learning society'. Syst. Res. Behav. Sci. 16(2), 113-122 (1999)

12. Lemoisson, P., Passouant, M.: Un cadre pour la construction collaborative de connaissances lors de la conception d'un observatoire des pratiques territoriales. Cah. Agric. 21(1), 11-17 (2012) 